\title{
New Traveling Waves for DNA's Vibrational Dynamics
}

\author{
Qian Lijuan ${ }^{1,3}$ \\ ${ }^{1}$ The Faculty of Science \\ Jiangsu University \\ Zhenjiang, Jiangsu, 212013,P.R.China \\ e-mail: lqian@csufresno.edu ; qlj@ujs.edu.cn \\ Tian Lixin ${ }^{2}$ \\ ${ }^{2}$ School of Mathematical Science \\ Nanjing Normal University \\ Nanjing, Jiangsu, 210024, P.R.China \\ e-mail: tianlx@ujs.edu.cn
}

\author{
Zhang Daming ${ }^{3}$ \\ ${ }^{3}$ Industrial Technology \\ California State University \\ Fresno, CA 93740-8002,USA \\ e-mail: dazhang@csufresno.edu
}

\begin{abstract}
In this paper, we focus on studying traveling waves for the nonlinear vibrational dynamics modeling DNA, which is modified from the Yakushevich model. The Yakushevich model of DNA torsion dynamics supports soliton solutions, which are supposed to be of special interest for DNA transcription. In the discussion of the model, we will use the traveling wave and a direct integral method, in special, take the integral constant to be some special value and a special change of variable to conclude some new explicit forms of traveling waves are given including smooth solitary waves, solitary waves with blow-up points, rational solutions with decay, periodic waves and polynomial solutions. While nonlocalized ones included periodic waves and polynomial solutions. At the same time, we give the figure of the smooth solitary, solitary waves with blow-up points, rational solution with decay and periodic waves. Those obtained new waves might show some special phenomena in transporting energy.
\end{abstract}

Keywords-component; DNA dynamics; Traveling waves; Smooth solitary waves

\section{INTRODUCTION}

The investigation of DNA dynamics via continuous and discrete models has successfully predicted the appearance of important nonlinear structures. It has also been shown that nonlinearity is responsible for forming localized waves. These localized waves are extremely interesting because they have the capability to transport energy without any dissipation [1-8].

Recently, there are several works about DNA dynamics. In [9] Cadoni M, De Leo R and Gaeta G propose and investigate, both analytically and numerically, a "composite" version of the Y model, in which the sugarphosphate group and the base are described by separate degrees of freedom. Peng XF focused on The properties of bio-energy transport and influence of structure nonuniformity and temperature of systems on energy transport along polypeptide chains [10]. J. D. Bashford [11]did some research for the sequence-dependent behaviour of localised excitations in a toy, nonlinear model of DNA base-pair opening originally proposed by Salerno. Specifically for whether "breather" solitons could play a role in the facilitated location of promoters by RNA polymerase (RNAP). For the inhomogeneous, DNA double helical molecular chain with flexible strands is investigated by studying its internal dynamics in [12] For the study, a generalized model which takes into account the energies involved in stacking and hydrogen bonds along with inhomogeneity, helicity, and phonons coupled to the stacking and hydrogen bonds is proposed. By using Riccati parameterized factorization method, W.Alka, Amit Goyal, C.Nagaraja. Kumar[13] studied the nonlinear dynamics of DNA, for longitudinal and transverse motions, in the framework of the microscopic model of Peyrard and Bishop, which consists of two long elastic homogeneous strands connected with each other by an elastic membrane. The improved Dauxoi-Peyrard-Bishop model for DNA as the simplest one for describing the appearance of nonlinear structures was analyzed by A Aguero and Jose Pecina in [14], and obtained new nonclassical traveling wave solutions i.e. anti-cuspon and peakon for specific restricted parametric values of the model. What about the propagation of a soliton-like excitation in a DNA was investigated through numerical integration of the motion equations[15], it showed that discreteness can completely change the soliton shape and the impact of viscosity as well as elasticity on DNA dynamic is also presented.

In [16], Daniel M,Vanitha $\mathrm{M}$ studied the internal nonlinear dynamics of an inhomogeneous short lattice DNA model, which is expressed in terms of open-state configurations represented by kink and antikink solitons with fluctuations, by solving numerically the governing discrete perturbed sine-Gordon equations under the limits of a uniform and a nonuniform angular rotation of bases. 
What about the Perturbed soliton excitations of the nonlinear dynamics of the inhomogeneous DNA doublehelical chain was studied in [17], by using the dynamic plane-base rotator model by considering angular rotation of bases in a plane normal to the helical axis. And inspired by [17], V Vasumathi and $M$ Daniel obtained the conclusions about perturbed solution-like molecular excitations in a deformed DNA chain in [18] .By an analysis of a multiple scale soliton perturbation, it solved the perturbed sine-Gordon equation and the resultant perturbed kink and antikink solitons represent open state configuration with small fluctuation. The perturbation due to periodic deformation of the lattice changes the velocity of the soliton, but the width remains unchanged. Slobodan Zdravkovic and Miljko V. Sataric studied a possible solitary wave solution of the nonlinear Schrödinger equation (NLSE) and DNA dynamics in [19], in which it was shown that the wave can be both modulated and nonmodulated depending on a ratio of the envelope and the carrier wave velocities.

In order to study some important aspects of DNA at the scale of base pairs, the oscillator-chain PB model proposed by Peyrard and Bishop has successfully predicted the appearance of solitonic structures [7]. Taking into consideration the inharmonic potential, Aguero et al. gave and studied the modified PB model [1] formed asThe paper is to be written in two-column format and be right and left justified. The column width should be $85 \mathrm{~mm}$ (3.35 inches). The gap between the two columns should be $5 \mathrm{~mm}$ ( 0.2 inches).

(1) $y_{t t}-\left[C_{1}+3 C_{2} y_{x}^{2}\right] y_{x x}-2 \alpha D e^{-\alpha y}\left(e^{-\alpha y}-1\right)=0$ where $C_{1}, C_{2}, D$ and $\alpha$ are constants. By a direct integral method, taking the integral constant to be some special value and a special change of variable, Aguero et al. obtained some nonclassical solution.

It is clear that the results in [1] are incomplete for the modified PB model. The purpose of this paper lies in a more complete study of the explicit traveling wave solutions. In this paper, by considering integral constants different from [1], we fortunately obtain some new explicit

traveling wave solutions, including localized waves and nonlocalized ones.

This paper is organized as follows. In Section 2, some new explicit forms of traveling waves are given by a direct integral method. Last section is the conclusion.

\section{NEW TRAVELING WAVES}

For a traveling wave $S=X-V_{t}$, Eq.(1) takes the form after integrating once

(2)

$$
\frac{V^{2}-C_{1}}{2} y_{s}^{2}-\frac{3}{4} C_{2} y_{s}^{4}+D e^{-\alpha y}\left(e^{-\alpha y}-2\right)+C=0
$$

where $C$ is an arbitrary integration constant. In order to simplify Eq.(2), we need to redefine the variables as follows

(3)

$$
\phi=e^{-\alpha y}
$$

Clearly, the fuction $\phi$ is positive. Hence we have $y=-\frac{1}{\alpha} \ln \phi$ and $y_{s}=-\frac{\phi_{s}}{\alpha \phi}$.Substituting $y \quad$ and $y_{s}$ into (2), we obtain

(4) $A \phi_{s}^{4}-B \phi_{s}^{2} \phi^{2}-D \phi^{5}(\phi-2)-C \phi^{4}=0$,

where $A=\frac{3}{4 \alpha^{4}} C_{2}$ and $B=\frac{V^{2}-C_{1}}{2 \alpha^{2}}$.

Simplifying (4) lets to

(5) $\phi_{s}^{2}=\frac{\phi^{2}}{2}\left(a+k \sqrt{4 b(\phi-1)^{2}+a^{2}-4 b+4 c}\right)$

where $k= \pm 1, \alpha=\frac{B}{A}, b=\frac{D}{A}$ and $c=\frac{C}{A}$

Letting $a^{2}-4 b+4 c=0$, that is, taking an integral constant $D=\frac{B^{2}}{4 A}+C$ which is not the same as [1], then (5) becomes

(6) $\phi_{s}^{2}=\phi^{2}\left(\frac{a}{2}-k \sqrt{b}+k \sqrt{b} \phi\right)$

By considering the different values of $a, b, k$ and noting the fact that $\phi$ is positive, we can obtain a series of fundamental solutions of Eq.(6) as follows.

If $b=0$, we have $\phi=\lambda \exp \left[ \pm \sqrt{\frac{a}{2}}\left(s-s_{0}\right)\right]$ where $\lambda>0$.

$$
\begin{gathered}
\text { If } \frac{a}{2}-k \sqrt{b}>0 \text { and } b>0 \text {, we have } \\
\phi=\frac{a}{2 \sqrt{b}}+1+\sec h^{2}\left[\frac{1}{2} \sqrt{\frac{a}{2}+\sqrt{b}}\left(s-s_{0}\right)\right] . \\
\text { If } \frac{a}{2}-k \sqrt{b}>0 \text { and } b>0 \text {, we can also obtain } \\
\phi=\frac{a}{2 \sqrt{b}}-1+\csc h^{2}\left[\frac{1}{2} \sqrt{\frac{a}{2}-\sqrt{b}}\left(s-s_{0}\right)\right] . \\
\quad \text { If } \frac{a}{2}-k \sqrt{b}=0 \quad \text { and } b>0 \text {,we have } \\
\phi=\frac{\sqrt{\frac{\alpha}{8}}\left(s-s_{0}\right)^{-2} .}{\text { If } \frac{a}{2}-k \sqrt{b}<0 \quad \text { and } b>0, \text { we have }} \\
\phi=-\frac{a}{2 \sqrt{b}}+1+\sec ^{2}\left[\frac{1}{2} \sqrt{\sqrt{b}-\frac{a}{2}}\left(s-s_{0}\right)\right] .
\end{gathered}
$$


By Eq.(3) and the above analysis, we find that Eq. (1) possesses some new traveling waves, which are classified as follows.

- $\quad$ Case A

If $D=0$, Eq.(1) possesses the polynomial solutions

$$
y=-\frac{\mu}{\alpha} \pm \frac{1}{\alpha} \sqrt{\frac{B}{2 A}}\left(s-s_{0}\right)
$$

where $\mu$ is an arbitrary constant.

- $\quad$ Case B

$$
\text { If } \frac{B}{2 A}+\sqrt{\frac{D}{A}}>0 \text { and } D>0 \text {, Eq. (1) admits the }
$$
smooth solitary waves

$$
y=-\frac{1}{\alpha} \ln \left\{\frac{B}{2 \sqrt{A D}}+1+\sec h^{2}\left[\frac{1}{2} \sqrt{\frac{B}{2 A}+\sqrt{\frac{D}{A}}}\left(s-s_{0}\right)\right]\right\} \text { If } \frac{B}{2 A}-\sqrt{\frac{D}{A}}=0 \text { and } D>0 \text {,Eq. (1) }
$$

Its 2-dimension graph when $t=0$ is shown in Fig.1 with $B=\alpha=A=D=1, s_{0}=0$.

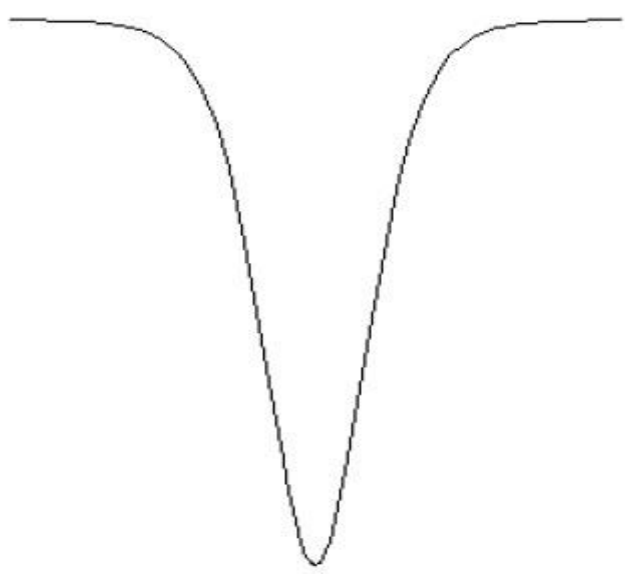

Figure 1. Smooth solitary waves

- $\quad$ Case C

If $\frac{B}{2 A}-\sqrt{\frac{D}{A}}>0$ and $D>0$, Eq. (1)

admits the solitary waves with blow-up points

$$
y=-\frac{1}{\alpha} \ln \left\{\frac{B}{2 \sqrt{A D}}-1+\csc h^{2}\left[\frac{1}{2} \sqrt{\frac{B}{2 A}-\sqrt{\frac{D}{A}}}\left(s-s_{0}\right)\right]\right\}
$$

- $\quad$ Case E

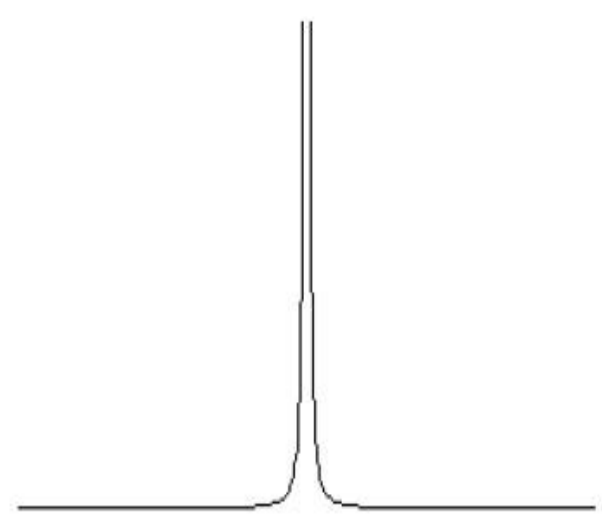

Figure 3.Rational solutions with decay admits the rational solutions with decay, $y=\sqrt{\frac{\alpha}{8}}\left(s-s_{0}\right)^{-2}$

Its 2-dimension graph when $t=0$ is shown in Fig.3 with $\alpha=1, s_{0}=0$.

Its 2-dimension graph when $t=0$ is shown in Fig.2 with $B=3, \alpha=A=D=1, s_{0}=0$ periodic waves

$y=-\frac{1}{\alpha} \ln \left\{\frac{B}{2 \sqrt{A D}}+1+\sec ^{2}\left[\frac{1}{2} \sqrt{-\frac{B}{2 A}+\sqrt{\frac{D}{A}}}\left(s-s_{0}\right)\right]\right\}$

Its 2-dimension graph when $t=0$ is shown in Fig.4 with $B=-3, \alpha=A=D=1, s_{0}=0$ 


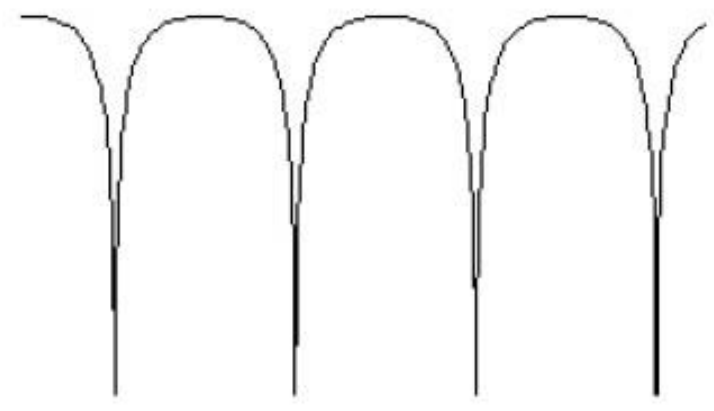

Figure 4.periodic waves

\section{CONCLUSIONS}

By a direct integral method, some localized traveling waves and nonlocalized traveling waves for the modified PB model were determined. Those localized waves included smooth solitary waves, solitary waves with blow-up points and rational solutions with decay. While nonlocalized ones included periodic waves and polynomial solutions. Those obtained new waves might show some special phenomena in transporting energy.

\section{ACKNOWLEDGMENT}

The work is supported by the National Natural Science Foundation of China (Program No. 11171135). The authors would also like to thank China Jiangsu University, and California University, Fresno for the support to the first author as a visiting scholar.

\section{REFERENCES}

[1] M.Aguero, M.Najera, M.Carrillo, "NONCLASSIC SOLITONIC STRUCTURES IN DNA's VIBRATIONAL DYNAMICS" , International Journal of Modern, Physics B, Vol 22, No 16; 25712582, (2008)

[2] G. Gaeta, "Results and limits of the soliton theory of DNA transcription", J.Biol. Phys ,Vol.24; 81-96(1999)
[3] G. Gaeta, C. Reiss, M. Peyrard and T. Dauxois. "Simple model of Non-linear DNA dynamics", Rivista del Nuovo Cimento, Vol. 17, No. 4, pp. 1-18, (1994)

[4] L. V. Yakushevich, "The effects of damping external fields and inhomogeneity on the nonlinear dynamics of biopolymers", Studia Biophys., Vol. 121, pp. 201-207, (1987).

[5] Yakushevich, L.V., Nonlinear DNA dynamics: a new model, Phys. Lett. A,Vol. 136 , Iss. 7-8, pp. 413-417, (1989).

[6] L. V. Yakushevich, Nonlinear Physics of DNA ,Wiley and Sons, England, 1998

[7] M. Peyrard and A. Bishop, "Statistical mechanics of a nonlinear model for DNA denaturation", Phys. Rev. Lett., Volume 62, Issue 23, June 5, 1989, pp.2755-2758.

[8] S. Zdravković and M. V. Satarić, "Single-molecule unzippering experiments on DNA and Peyrard-Bishop-Dauxois model", Phys. Rev. E Vol. 73, pp. 1-11, (2006).

[9] Cadoni M, De Leo R, Gaeta G. "Composite model for DNA torsion dynamics". Phys Rev E Stat Nonlin Soft Matter Phys. 2007 Feb;75(2 Pt 1):021919. Epub 2007 Feb 28.

[10] Pang XF. "The properties of bio-energy transport and influence of structure nonuniformity and temperature of systems on energy transport along polypeptide chains". Prog Biophys Mol Biol (2012)108(1-2) pp :1-46

[11] J. D. Bashford. "Salerno's Model of DNA Re-Analysed: Could Breather Solitons have Biological Significance?" J Biol Phys 32(1), 2006, pp: 27-47.

[12] Daniel M,Vanitha M. "Bubble solitons in an inhomogeneous, helical DNA molecular chain with flexible strands", Phys Rev E Stat Nonlin Soft Matter Phys. 2011 Sep;84(3 Pt 1):031928. Epub 2011 Sep 27

[13] W.Alka, Amit Goyal, C.Nagaraja. Kumar. "Nonlinear dynamics of DNA - Riccati generalized solitary wave solutions",Physics Letters A 375(3),2011,pp:480-483.

[14] A Aguero, Jose Pecina. "Peakon and cuspon as collective excitations in nonlinear dynamics of DNA" , Physica Scripta 06/2010; 82(1):015009

[15] Tabi CB, Mohamadou A, Koofane TC Eason. "Soliton-like excitation in a nonlinear model of DNA dynamics with viscosity". Math Biosci Eng. 2008 Jan;5(1):205-216.

[16] Daniel M,Vanitha M. "Internal nonlinear dynamics of a shor lattice DNA model in terms of propagating kink-antikink solitons." Phys Rev E Stat Nonlin Soft Matter Phys. 2012 Apr;85(4 Pt 1):041911. Epub 2012 Apr 13.

[17] M Daniel, V Vasumathi. "Perturbed solition excitations in the DNA double helix.” Physical D: Nonlinear Phenomena, Vol 231,Issue 1, 2007,pp: 10-29.

[18] V Vasumathi, M Daniel. "Perturbed Solution-like Molecular Excitations in a Deformed DNA Chain." Physics Letters A, Vol 373, Issue 1, 2008, pp: 76-82

[19] Slobodan Zdravkovic and Miljko V. Sataric. "Nonlinear Schrödinger Equation (NLSE) and DNA Dynamics.” Physics Letters A, Vol 373, Issue 1, 2008, pp: 126-132. 